\title{
Semaglutid - Pharmakodynamische und pharmakokinetische Eigenschaften eines neuen lang wirksamen GLP-1-Rezeptoragonisten
}

\section{Semaglutide - Pharmacodynamic and Pharmacokinetic Characteristics of a New Long-Acting GLP-1-Receptor Agonist}

Autoren

Michael A. Nauck'1, Baptist Gallwitz² ${ }^{2}$ Jochen Seufert ${ }^{3}$

Institute

1 Diabetologie, St. Josef-Hospital (Ruhr-University Bochum), Bochum, Germany

2 Medizinische Klinik IV, Medizinische Klinik Tübingen, Germany

3 Abteilung Endokrinologie und Diabetologie, Klinik für Innere Medizin II des Universitätsklinikums Freiburg, Medizinische Fakultät, Albert-Ludwigs-Universität Freiburg, Germany

Schlüsselwörter

Semaglutid, GLP-1-Rezeptoragonisten, Pharmakodynamik, Pharmakokinetik, Halbwertszeit, Wechselwirkungen

Key words

semaglutide, GLP-1 receptor agonists, pharmacodynamics, pharmacokinetics, half-life period, drug-drug interactions

eingereicht 27.12.2016

akzeptiert 27.02.2017

Bibliografie

DOI http://dx.doi.org/10.1055/s-0043-104866

Online-Publikation: 22.3.2017 | Diabetologie 2017; 12: 135140 @ Georg Thieme Verlag KG Stuttgart · New York ISSN 1861-9002

Korrespondenzadresse

Michael A. Nauck

Diabetologie, St. Josef-Hospital (Ruhr-University Bochum)

Gudrunstraße 56

D-44791 Bochum

Germany

Tel.: ++ 492345096332

michael.nauck@rub.de

\section{ZUSAMMENFASSUNG}

Semaglutid ist ein GLP-1-Rezeptoragonist, der eine $94 \%$ ige Aminosäuresequenz-Homologie zu humanem GLP-1 aufweist. Aufgrund von Molekülmodifikationen bindet es nach subkutaner Gabe stark an Plasmaalbumin und weist eine sehr lange Halbwertszeit von 165 bis 200 Stunden auf. Dies ermöglicht eine einmal wöchentliche subkutane Gabe.

Phase-1- und Phase-2-Studien haben ergeben, dass Semaglutid die typischen Wirkungen eines GLP-1-Analogons zeigt, wie Aktivierung der Insulinfreisetzung und Hemmung der Glucagonsekretion. Die für alle GLP-1-Rezeptoragonisten typischen Nebenwirkungen Übelkeit, Brechreiz und Diarrhoe treten auch unter Semaglutid dosisabhängig auf, nehmen aber im weiteren Verlauf der Therapie an Häufigkeit und Schwere ab. Pharmakokinetische Untersuchungen an gesunden Probanden und Patienten mit Typ-2-Diabetes belegen die lange anhaltende, gleichmäßige Exposition mit einmal wöchentlicher Gabe. Semaglutid beeinflusst nicht die Plasmakonzentrationen von gleichzeitig verabreichtem Metformin, Warfarin, Atorvastatin, Digoxin, Ethinylestradiol oder Levonorgestrel. Ergebnisse des Phase-3-Studienprogramms bei Patienten mit Typ 2-Diabetes werden in einem eigens der Phase 3 gewidmeten Artikel vorgestellt [1].

\section{ABSTRACT}

Semaglutide is a GLP-1 agonist, which shows a $94 \%$ amino acid homology to human GLP-1. Due to molecular modifications, Semaglutide binds after s. c. injection strongly to plasma albumin. It has a very long half-life of approximately 165 to 200 hours, which permits once-weekly subcutaneous administration.

Phase 1 and Phase 2 studies have shown, that semaglutide is acting like a typical GLP-1 analogue. It activates insulin release and inhibits glucagon secretion. Side effects as nausea, vomiting, and diarrhea, typical for GLP-1 agonists, are occurring in a dose-dependent manner with Semaglutide too, but frequency and severity are declining with ongoing therapy. Pharmacokinetic and pharmacodynamic studies prove a sustained and constant exposition with once weekly administration. Semaglutide has no effect on plasma concentration of simultaneously administered metformin, warfarin, atorvastatin, digoxine, ethinylestradiol or levonorgestrel.

Results of the phase 3 program in patients with type 2 diabetes are presented in a separate, dedicated manuscript [1]. 
- Tab. 1 Effekte von kurz und lang wirkenden GLP-1-Rezeptoragonisten. Mod. nach [2].

\begin{tabular}{|l|l|l|}
\hline Effekte & Kurz wirksame GLP-1 RA & Lang wirksame GLP-1 RA \\
\hline Substanzen & Exenatid BID, Lixisenatid & $\begin{array}{l}\text { Liraglutid, Exenatid QW, Dulaglutid, Albiglutid, } \\
\text { Semaglutid }\end{array}$ \\
\hline Nüchternblutzuckerwerte & Geringe Reduktion & Starke Reduktion \\
\hline Postprandiale Hyperglykämie & $\begin{array}{l}\text { Ausgeprägte Reduktion nach Mahlzeiten, vor } \\
\text { denen das Medikament verabreicht wurde }\end{array}$ & Geringe Reduktion \\
\hline Nüchterninsulinsekretion & Geringe Stimulation & Starke Stimulation \\
\hline Glucaconsekretion & Reduktion & Reduktion \\
\hline Magenentleerung & Verzögerung & Vorübergehende Verzögerung (Tachyphylaxie) \\
\hline Gewichtsreduktion & Variabel, im Mittel 2-4 kg & Variabel, im Mittel 2-4kg \\
\hline Blutdruck & Reduktion & Reduktion \\
\hline Herzfrequenz & Anstieg bei Medikamentenexposition & Anstieg (dauerhaft) \\
\hline
\end{tabular}

\section{Einleitung}

Glucagon-like Peptide 1 (GLP-1) ist ein Inkretinhormon, das im Magen-Darm-Trakt gebildet wird und die Glucagonsekretion in Abhängigkeit von der Glucosekonzentration im Blut hemmt sowie die Insulinsekretion aktiviert. Es verzögert zudem die Magenentleerung und verringert den Appetit und somit die Nahrungsaufnahme [2]. Diese Wirkungen sind auch bei Patienten mit Typ-2Diabetes nachweisbar und führen bei pharmakologischer Dosierung zur Senkung erhöhter Blutzuckerkonzentrationen. Daher ist GLP-1 eine geeignete Muttersubstanz für blutzuckersenkende Medikamente für die Therapie des Diabetes mellitus Typ 2. Geeignete GLP-1-Derivate, sogenannte GLP-1-Rezeptoragonisten (GLP1 RA), haben sich mittlerweile in der Therapie von Patienten mit Diabetes mellitus Typ 2 etabliert [3, 4]. Eingesetzt werden kurz wirkende Substanzen wie Exenatid (s. c. Injektion zweimal täglich) oder Lixisenatid (s. c. Injektion einmal täglich), die jeweils nur vorübergehend für einige Stunden zu wirksamen Medikamentenkonzentrationen führen. Dauerhaft erhöhte Medikamentenkonzentrationen erzielen lang wirksame GLP-1-Rezeptoragonisten wie Liraglutid (einmal tägliche s.c. Injektion) und langwirksames Exenatid sowie Albiglutid, Dulaglutid und Semaglutid (einmal wöchentliche s. c. Injektion) [5].

\section{Wirken alle GLP-1-Analoga gleich?}

Während das grundlegende Wirkprofil (auf Insulin- und Glucagonsekretion, Magenentleerung und Appetitregulation) im Wesentlichen vergleichbar ist, sind zwischen kurz und lang wirksamen GLP-1 RA die klinischen Effekte unterschiedlich gewichtet ( $\triangleright$ Tab. 1): Kurz wirksame GLP-1 RA senken wegen eines dauerhaft erhaltenen Effekts auf die Magenentleerung ausgeprägter postprandiale Blutzuckeranstiege nach der Mahlzeit, vor der sie injiziert worden sind, während lang wirksame GLP-1 RA einen ausgeprägteren Effekt auf den Nüchternblutzucker haben [6]. Diese unterschiedlichen Eigenschaften ermöglichen eine individualisier- te Therapie des Patienten mit Diabetes mellitus Typ 2 [2], z. B. in Abhängigkeit von einer gleichzeitigen Therapie mit Basalinsulin.

\section{Das GLP-1-Analogon Semaglutid}

Mit Semaglutid (Novo Nordisk) wurde ein neues, lang wirkendes GLP-1-Analogon entwickelt. Aktuelle Ergebnisse des Phase-3-Programms (SUSTAIN: Semaglutide Unabated Sustainability in Treatment of Type 2 Diabetes) belegen die ausgeprägten Blutzuckerund Gewichts- senkenden Effekte von Semaglutid bei Patienten mit Typ-2-Diabetes mellitus bei einem mit anderen langwirksamen GLP-1 RA vergleichbaren Verträglichkeitsprofil. Zudem konnte in der SUSTAIN-6-Studie gezeigt werden, dass Semaglutid bei Patienten mit Typ-2-Diabetes und besonders hohem kardiovaskulärem Risiko zusätzlich zur Senkung des Blutzuckerspiegels auch die Rate kardiovaskulärer Ereignisse signifikant verringern konnte, allerdings wurde das Risiko für eine Retinopathie bzw. für Komplikationen einer Retinopathie im Vergleich zu Placebo erhöht [1, 7].

Schwerpunkt des vorliegenden Übersichtsartikels sollen die pharmakokinetischen und pharmakodynamischen Eigenschaften von Semaglutid sein.

\section{Molekülstruktur von Semaglutid}

Strukturell ist Semaglutid eng mit Liraglutid verwandt. Es ist dem menschlichen GLP-1-(7 - 37) mit einer Aminosäuresequenz-Homologie von $94 \%$ sehr ähnlich [8] und unterscheidet sich von der Muttersubstanz durch folgende Molekülmodifikationen

$(\vee$ Abb. 1):

- Substitution der Aminosäure Alanin in Position 8 durch die synthetische Aminosäure Alpha-Amino-Iso-Buttersäure, die Semaglutid vor dem Abbau durch DPP-4 schützt

- Acylierung des Peptid-Grundgerüsts mit einer 18-gliedrigen Dicarbonsäure am Lysin in Position 26 über einen synthetischen Spacer. Dadurch bindet sich das Molekül stark an Albu- 




min, was die renale Clearance verringert und die Halbwertszeit verlängert [8, 9].

- Substitution der Aminosäure Lysin in Position 34 durch Arginin verhindert die Bindung der C18-Dicarbonsäure an der falschen Stelle.

\section{Wirkungen von Semaglutid}

Semaglutid ist ein Inkretinmimetikum, das an GLP-1-Rezeptoren bindet und dosisabhängig insulinotrop wirkt. Im Vergleich zu Liraglutid hat es in vitro eine ähnlich gute Affinität zu GLP-1-Rezeptoren, jedoch auch eine hohe Affinität zu Albumin, was zu einer Reservoirbildung in Form von albumingebundenem Semaglutid führt und so die Verweildauer im Organismus verlängert [5].

\section{Pharmakokinetik von Semaglutid}

Die pharmakokinetischen Untersuchungen mit Semaglutid bei Probanden und bei Patienten mit Diabetes mellitus Typ 2 zeigen, dass der GLP-1-Rezeptoragonist nach subkutaner Gabe etwa 24 $36 \mathrm{~h}$ bis zum Erreichen der höchsten Konzentration benötigt. Aufgrund seiner hohen Bindung an Plasmaalbumin wird über lange Zeit eine hohe Plasmakonzentration aufrechterhalten. Die Plasmahalbwertszeit liegt zwischen 165 und 200 Stunden. Dies ermöglicht eine einmal wöchentliche Gabe [10 - 12] ( $\triangleright$ Abb. 2).

Semaglutid wird stark metabolisiert und die inaktiven Metaboliten werden renal und über den Faeces eliminiert. Im Urin findet sich dagegen nur wenig unverändertes Semaglutid [12].

\section{Dosisfindungsstudie bei Probanden}

Bei gesunden Männern führte Semaglutid, in steigenden Einzeldosen verabreicht [10], zu einer signifikanten Senkung des Nüchternblutzuckers ( $p=0,013)$, einer Steigerung der Nüchterninsulinkonzentrationen $(p<0,001)$ und einer Senkung der Nüchternglucagonkonzentrationen $(p=0,04)$ an Tag 2 nach s. c. Injektion. Mit visuellen Analogskalen wurden ein reduziertes Hungergefühl und ein vermehrtes Sättigungsgefühl nachgewiesen [10].

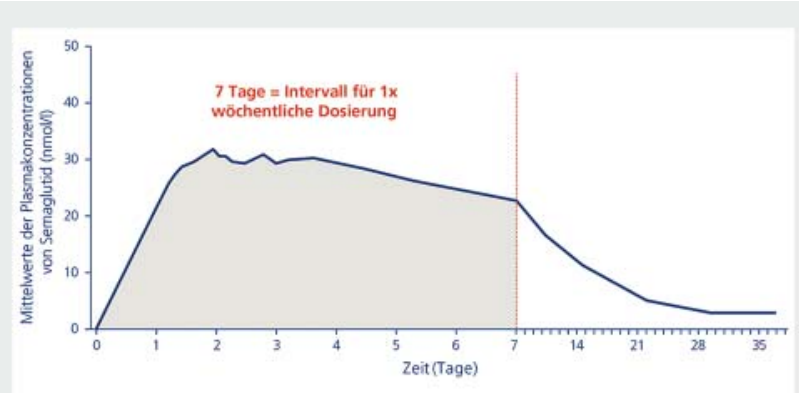

Im Steady State hat Semaglutid $1,0 \mathrm{mg}$ eine mediane $t_{\max }$ von 36 Stunden und eine mittlere $t_{1 / 2}$ von 165 Stunden

- Abb. 2 Mittlere Plasmakonzentration von Semaglutid im SteadyState bei Patient mit Diabetes mellitus Typ 2, die wiederholt Semaglutid einmal wöchentlich injiziert hatten. Verändert nach [8].

\section{Wirkung auf Appetit und Energiezufuhr, Glucose- und Fettstoffwechsel bei Probanden}

In einer randomisierten doppelblinden placebokontrollierten Crossover-Studie über zweimal zwölf Wochen mit 30 Männern und Frauen, jeweils mit einem Körpermassenindex (BMI) zwischen 30 und $45 \mathrm{~kg} / \mathrm{m}^{2}$ und einem $\mathrm{HbA}_{1 \mathrm{c}}$ unter $6,5 \%$, aßen die Probanden nach Behandlung mit Semaglutid im Vergleich zu Placebo weniger. Ein Nüchtern-Appetit-Score (visuelle Analogskala) zeigte einen durch Semaglutid signifikant verminderten Appetit im Vergleich zu Placebo ( $p=0,0023)$. Nach den Ergebnissen des „Control of Eating Questionnaire“ hatten die Semaglutidprobanden weniger Hunger und weniger Verlangen nach Nahrung. Sie konnten ihr Essverhalten besser kontrollieren. Unter Semaglutid nahmen die Probanden um 5,0 $\pm 2,4 \mathrm{~kg}$ Körpergewicht ab, unter Placebo um 1,0 $\pm 2,4 \mathrm{~kg}$ zu [13].

Nach zwölf Wochen Behandlung waren Nüchternblutglucoseund Glucagonkonzentration unter Semaglutidtherapie signifikant niedriger als unter Placebo, während die Nüchternkonzentrationen von Insulin und C-Peptid signifikant höher waren. Nach einem Standardfrühstück stiegen bei Semaglutidbehandlung die postprandialen Glucose-, Insulin- und C-Peptidwerte signifikant weniger als unter Placebo.

Die Nüchterntriglycerid- und LDL-Lipoproteinspiegel waren nach zwölf Wochen Behandlung mit Semaglutid ebenfalls signifikant niedriger als mit Placebo. Nach einem Standardfrühstück stiegen Triglyceride, LDL-Lipoproteine und Apolipoprotein B48 (vermittelt die Fettabsorption im Darm) signifikant weniger stark als nach Placebo.

Auswirkungen auf die Magenentleerung wurden nach 12-wöchiger Behandlung anhand der Paracetamol-Konzentrations-ZeitKurve von 0 bis 300 Minuten nach einem Standardfrühstück gemessen. Sie wurde durch Semaglutid im Vergleich zu Placebo nur in der ersten Stunde nach dem Standardfrühstück verlangsamt. Insgesamt unterschied sich die postprandiale Magenentleerung nicht zwischen den beiden Gruppen [14]. Die nachlassende Wirkung auf die Magenentleerung beruht vermutlich auf einer Tachyphylaxie, die bei anhaltend hohen Konzentrationen von GLP-1 beobachtet werden kann [15]. 


\section{Dosisfindungsstudie bei Patienten mit Diabetes melli- tus Typ 2}

Ein einer multizentrischen randomisierten Phase-2-Studie über zwölf Wochen erhielten 415 Patienten mit Diabetes mellitus Typ 2 Semaglutid einmal wöchentlich, entweder ohne initiale Dosiseskalation $(0,1$ bis $0,8 \mathrm{mg}$ ) oder mit langsamer initialer Dosiseskalation (Schritte von $0,4 \mathrm{mg}$ bis 0,8 oder $1,6 \mathrm{mg}$ über ein bis zwei Wochen), alternativ Liraglutid einmal täglich (1,2 oder $1,8 \mathrm{mg}$ ) oder Placebo ( $\triangleright$ Abb.3). Die Studie wurde für den Vergleich von Semaglutid und Placebo doppelblind und für den Vergleich mit der aktiven Kontrolle Liraglutid offen durchgeführt. Primärer Endpunkt war die Änderung im $\mathrm{HbA}_{1 c}$ im Vergleich zum Ausgangswert. Zu den sekundären Endpunkten gehörten Änderungen im Körpergewicht sowie die Erhebung von Sicherheits- und Verträglichkeitsparametern [3].

Wie $>$ Abb. 4 zeigt, senkte Semaglutid den $\mathrm{HbA}_{1 \mathrm{c}}$ dosisabhängig ( $\mathbf{A b b}$. 4a). Bis zu 81 \% der Patienten erreichten einen $\mathrm{HbA}_{1 c^{-}}$ Wert unter 7,0\%. Das Körpergewicht sank um bis zu 4,8 kg mit $1,6 \mathrm{mg}$ Semaglutid (mit initialer Dosiseskalation $p<0,001$ ) im Vergleich zu Placebo ( $\triangleright$ Abb. 4b). Semaglutid verringerte den $\mathrm{HbA}_{1 c^{-}}$ Wert und das Körpergewicht stärker als Liraglutid 1,2 mg und $1,8 \mathrm{mg}[3]$.

Unerwünschte Wirkungen wie Übelkeit und Erbrechen nahmen mit steigender Semaglutiddosis zu. Die meisten unerwünschten Wirkungen waren leichter Natur und vorübergehend und besserten sich bei weiterer Dosiseskalation. Es wurden keine starken Hypoglykämien beobachtet, zu Reaktionen an der Injektionsstelle kam es nur in wenigen Fällen.

\section{Fazit aus der Phase-2-Studie}

Aufgrund der Ergebnisse der Dosisfindungsstudien wurde entschieden, Semaglutid in Phase-3-Studien in Dosierungen von 0,5 und $1,0 \mathrm{mg}$ zu untersuchen und vor Erreichen der angestrebten Dosis eine langsame Dosiseskalation vorzuschalten (nach vier Wochen von 0,25 auf $0,5 \mathrm{mg}$ und bei Bedarf von $0,5 \mathrm{mg}$ auf $1,0 \mathrm{mg}$ nach weiteren vier Wochen), weil sich dies als wirksam erwiesen hatte, Nebenwirkungen zu verhindern und eine höhere Erhaltungsdosis zu erreichen [3].

In der Tat konnte durch die Wahl geeigneter Dosierungen und eines vorsichtigen Dosiseskalationsschemas in den nachfolgenden Phase-3-Studien eine deutliche Verbesserung in Hinsicht auf "gastrointestinale“ Nebenwirkungen erreicht werden [1, 7].

\section{Wirkung von Semaglutid auf die Insulinsekretion bei Patienten mit Diabetes mellitus Typ 2}

In einer randomisierten, doppelblinden, placebokontrollierten Parallelgruppen-Studie wurden die Effekte von Semaglutid auf die In-vivo-Insulinsekretion bei Patienten mit Diabetes mellitus Typ 2 untersucht. Sie erhielten einmal wöchentlich Semaglutid subkutan bis zu einer maximalen Dosis von $1,0 \mathrm{mg}(\mathrm{n}=37)$ oder Placebo $(n=38)$ über zwölf Wochen [16].

Die Wirkungen von Semaglutid auf die erste (0 - $10 \mathrm{~min})$ und die zweite Phase (10 - $120 \mathrm{~min}$ ) der Insulinsekretion wurden mit einem intravenösen Glucosetoleranztest ( $25 \mathrm{~g}$ Bolus, i. v. verabreicht über $2 \mathrm{~min}$ ) untersucht. Die Anstiege von Insulin, aber

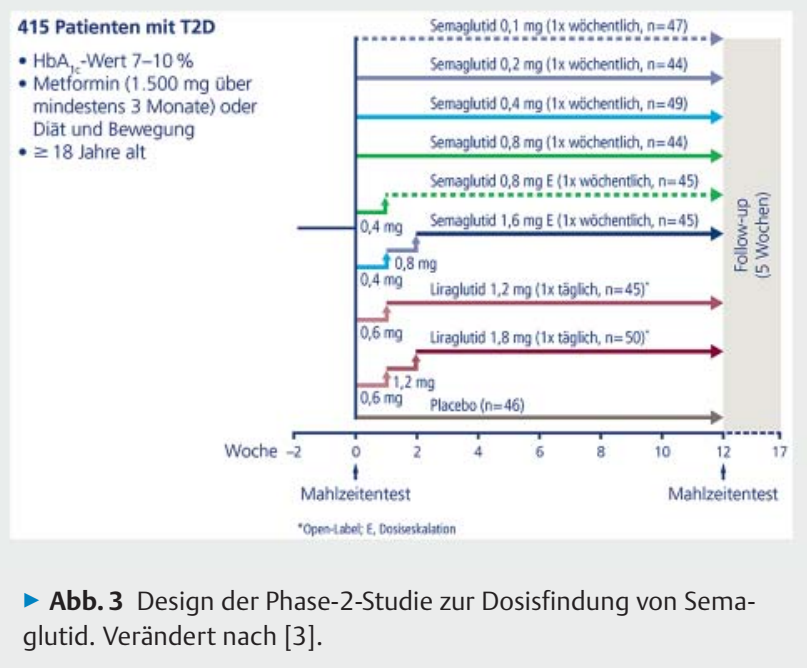

auch C-Peptid und kalkulierten Insulinsekretionsraten (Dekonvolution) in beiden Phasen waren nach zwölf Wochen Behandlung mit Semaglutid im Vergleich zu Placebo signifikant höher (jeweils $p \leq 0,0002$ ). Die Glucagonsekretion wurde unter Semaglutid signifikant verringert [16].

Das Ansprechen der pankreatischen Betazellen auf langsam steigende Blutzuckerkonzentrationen wurde mit einer abgestuften Glucoseinfusion untersucht. Nach Behandlung mit Semaglutid nahm die Insulinsekretionsrate im Vergleich zu Placebo signifikant zu, während die Glucagonkonzentrationen signifikant sanken. Der Verlauf der Parameter, die die Insulinsekretion abbilden, ähnelte bei Semaglutidtherapie dem von gesunden (unbehandelten) Probanden, unterschied sich jedoch deutlich von dem der Patienten mit Diabetes, die Placebo erhalten hatten.

Die Ergebnisse dieser Untersuchung weisen also darauf hin, dass eine zwölfwöchige Behandlung mit Semaglutid die Insulinsekretion im Vergleich zu Placebo bei Patienten mit Diabetes mellitus Typ 2 verbessert und dass unter der Therapie normale Muster (wie bei gesunden Probanden) beobachtet werden können [16].

\section{Wechselwirkungen mit Metformin, Warfarin, Atorva- statin, Digoxin und oralen Kontrazeptiva}

Bei gesunden Erwachsenen wurden die Effekte einer Semaglutidtherapie auf Pharmakokinetik von gleichzeitig verabreichtem Metformin, Warfarin, Atorvastatin und Digoxin untersucht. Der GLP-1-Rezeptoragonist veränderte bei keinem der gleichzeitig verabreichten Medikamente die pharmakokinetischen Parameter wie die Fläche unter der Serumkonzentrationszeitkurve oder die maximale Plasmakonzentration signifikant [17]. Wird Semaglutid also zusätzlich zu diesen Substanzen gegeben, sind keine Dosisanpassungen erforderlich.

In einer Crossover-Studie mit 43 postmenopausalen Frauen mit Diabetes mellitus Typ 2 wurden die Wechselwirkungen von Semaglutid mit oralen Kontrazeptiva untersucht. Die Ergebnisse zeigten, dass Semaglutid die Bioverfügbarkeit von Ethinylestradiol und Levonorgestrel nicht signifikant verringerte [8]. 

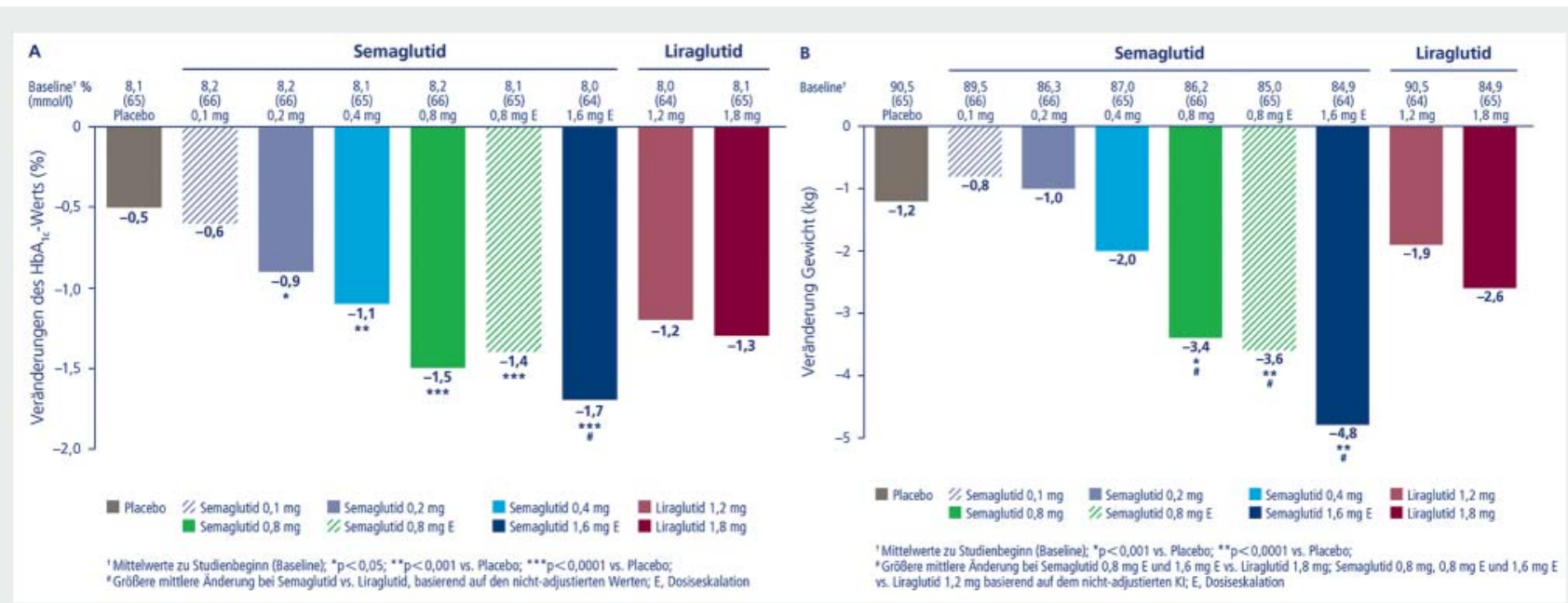

- Abb. 4 Senkung des $\mathrm{HbA}_{1 c}$-Werts $\mathbf{A}$ und des Körpergewichts $\mathbf{B}$ durch steigende Dosen von Semaglutid sowie durch Liraglutid bei Patienten mit Diabetes mellitus Typ 2. Verändert nach [3].

\section{Fazit für die Praxis}

Semaglutid ist ein lang wirkender GLP-1-Rezeptoragonist mit einer Halbwertszeit zwischen 165 und 200 Stunden, der einmal wöchentlich subkutan injiziert werden kann. Er zeigt die typischen Wirkungen eines lang wirkenden GLP-1-Analogons, nämlich Stimulation der Insulinsekretion und Hemmung der Glucagonfreisetzung sowie Hemmung von Appetit und Nahrungsaufnahme, was zu einer nennenswerten Blutzuckersenkung und einer ausgeprägten Gewichtsreduktion führt. Die als häufigste unerwünschte Wirkung auftretenden gastrointestinalen Störungen nehmen im Verlauf der Therapie ab.

Eine Dosisanpassung ist bei gleichzeitiger Gabe von Metformin, Warfarin, Atorvastatin und Digoxin nicht notwendig. Zudem wird die Bioverfügbarkeit von oralen Kontrazeptiva nicht signifikant beeinflusst.

Semaglutid ist aufgrund dieser Eigenschaften ein viel versprechendes neues Diabetesmedikament. Ergebnisse der klinischen Phase-3-Studien einschließlich einer kardiovaskulären „Outcomes"-Studie werden in einem begleitenden Artikel ausführlicher dargestellt [1].

\section{Danksagung}

Für die redaktionelle Mitarbeit danken wir Frau Dr. Susanne Heinzl, Reutlingen, die dafür eine Vergütung von Novo Nordisk erhalten hat.

\section{Interessenkonflikt}

Prof. Dr. med. Michael A. Nauck hat bei Advisory Boards mitgewirkt oder Berater-Honorare bezogen für Berlin Chemie, Amylin Pharmaceuticals, AstraZeneca, Boehringer Ingelheim, Eli Lilly \& Co., Fractyl, GlaxoSmithKline, MSD, Novo Nordisk, Sanofi, Intarcia; er hat Honorare für Vorträge von AstraZeneca, Berlin Chemie, Boehringer Ingelheim, Diabate, Eli Lilly \& Co., Incretin Expert Pro- gram, Medscape, MSD, Novartis, Novo Nordisk, erhalten, und Reise- und Verpflegungskosten im Rahmen der o. a. Aktivitäten wurden erstattet.

Prof. Dr. med. Baptist Gallwitz: Board Member/Advisory Panel: Amgen, AstraZeneca, Bristol Myers Squibb, Boehringer Ingelheim, Janssen, Lilly, Merck Sharp \& Dohme, Novartis, Novo Nordisk; Vortragshonorare: Abbott, Amgen, AstraZeneca, Bristol Myers Squibb, Boehringer-Ingelheim, Lilly, Merck Sharp \& Dohme, Novartis, Novo Nordisk, Sanofi.

Prof. Dr. med. Jochen Seufert hat Vortragshonorare und/oder Beraterhonorare und/oder Forschungsförderung (als zweckgebundene Drittmittel) von Apitope, Astra Zeneca, Bayer, Berlin Chemie, Boehringer Ingelheim, Bristol Myers Squibb (BMS), GIDynamics, Glaxo Smith Kline (GSK), Intarcia, Ipsen, Janssen, LifeScan, Lilly, Merck Sharp Dohme (MSD), MedScape, Novartis, NovoNordisk, Omniamed, Pfizer, Roche, Sanofi Aventis, Servier, Takeda, Ypsomed erhalten.

\section{Literatur}

[1] Seufert J, Nauck MA, Gallwitz B. Semaglutid - ein neuer langwirksamer GLP-1 RezeptorAgonist mit nachgewiesener kardiovaskulärer Ereignisreduktion bei Typ-2-Diabetes. Diabetologie Stoffw 2017; 12

[2] Meier JJ. GLP-1 receptor agonists for individualized treatment of type 2 diabetes mellitus. Nature Rev Endocrinol 2012; 8: 728-742

[3] Nauck MA, Petrie JR, Sesti G et al. A phase 2, randomized, dose-finding study of the novel once-weekly human GLP-1 analog, semaglutide, compared with placebo and open-label liraglutide in patients with type 2 diabetes. Diabetes Care 2016; 39: 231 - 241

[4] Nauck M. Incretin therapies: highlighting common features and differences in the modes of action of glucagon-like peptide- 1 receptor agonists and dipeptidyl peptidase-4 inhibitors. Diabetes Obes Metab 2016; 18: $203-216$

[5] Lau J, Bloch P, Schaffer L et al. Discovery of the once-weekly glucagonlike peptide-1 (GLP-1) analogue semaglutide. J Med Chem 2015; 58: $7370-7380$ 
[6] Fineman MS, Cirincione BB, Maggs D et al. GLP-1 based therapies: differential effects on fasting and postprandial glucose. Diabetes Obes Metab 2012; 14: 675-688

[7] Marso SP, Bain SC, Consoli A et al. Semaglutide and cardiovascular outcomes in patients with type 2 diabetes. N Engl J Med 2016; 375: 1834 1844

[8] Kapitza C, Nosek L, Jensen L et al. Semaglutide, a once-weekly human GLP-1 analog, does not reduce the bioavailability of the combined oral contraceptive, ethinylestradiol/levonorgestrel. J Clin Pharmacol 2015; 55: $497-504$

[9] Lorenz M, Evers A, Wagner M. Recent progress and future options in the development of GLP-1 receptor agonists for the treatment of diabesity. Bioorg Med Chem Lett 2013; 23: 4011-4018

[10] Kapitza C, Lynge J, Düring M et al. Safety, tolerability, pharmacokinetics (PK) pharmacodynamics (PD) of single escalating doses of semaglutide, a unique once weekly GLP-1 analogue, in healthy male subjects. Diabetologia 2012; 55: S341

[11] Marbury T, Flint A, Segel $S$ et al. Pharmacokinetics and tolerability of a single dose of semaglutide, a once-weekly human GLP-1 analogue, in subjects with and without renal impairment. Diabetes 2014; 63 (Suppl. 1): A260 1010-P
[12] Roffel AF, Jensen L, van Lier II et al. Absorption, metabolism and excretion of $[3 \mathrm{H}]$-semaglutide in healthy male subjects. Diabetologia 2015; 58 (Suppl. 1): Poster 0225-P

[13] Blundell ], Finlayson G, Axelsen MB et al. Semaglutide reduces appetite and energy intake, improves control of eating, and provides weight loss in subjects with obesity. Diabetes 2016; 65 (Suppl. 1): A6

[14] Hjerpsted J, Axelsen MB, Brooks A. Semaglutide improves postprandial glucose and lipid metabolism and delays first-hour gastric emptying in subjects with obesity. Diabetes 2016; 65 (Suppl. 1): A273 1046-P

[15] Nauck MA, Kemmeries G, Holst JJ et al. Rapid tachyphylaxis of the glucagon-like peptide 1-induced deceleration of gastric emptying in humans. Diabetes 2011; 60: 1561 - 1565

[16] Kapitza C, Dahl K, Jacobsen JB et al. The effects of once-weekly semaglutide on B-cell function in subjects with type 2 diabetes. Diabetologia 2016; 59 (Suppl. 1): Poster \#754

[17] Hausner H, Anderson TW, Karsbol JD et al. Effect of once weekly subcutaneous treatment with semaglutide, a GLP1 Analog, on metformin, warfarin, atorvastatin and digoxin in healthy subjects. 98th Annual Meeting Endocrine Society. Boston, 2016. FRI-710 